\title{
Niemand weiß, was Gesundheit ist - deshalb haben wir Gesundheitsprobleme?
}

Zum Editorial: Große K, Rendenbach U. Gesundheit - eine Reform des Begriffs? Notfall \& Hausarztmedizin 2008; 34 (7): 347

Die gewählte Überschrift unseres Beitrags bestätigt in gewisser Weise den von Große und Rendenbach für die USA aufgezeigten Wellness-Trend zu einer „ÜberGesundheit“. Gesund, gesünder, am gesündesten! Dabei sollte Gesundheit eigentlich ein Optimum sein. Die häufig in Anspruch genommene WHO-Gesundheitscharakteristik „Die Gesundheit ist der Zustand des vollständigen, körperlichen, geistigen und sozialen Wohlbefindens und nicht nur das Freisein von Kranksein und Gebrechen“ ist als zeitbedingte politische Deklaration zu interpretieren, keinesfalls aber mit einer Definition $\mathrm{zu}$ verwechseln. Letztere sind ebenso zahlreich wie unterschiedlich; ein Indiz dafür, dass eine hinreichende allseits akzeptierte und anerkannte, theoretische Begründung dieser Grundkategorie der Medizin noch immer aussteht. Auch der Begriff der Normalität im Sinne des statistischen Durchschnitts bietet keinen Beleg für Gesundheit, wenn beispielsweise Wirth feststellt: „In Deutschland ist es schon normal, nicht mehr normalgewichtig zu sein“ (1).

Gesundheit ist weder Zustand noch Besitzgut, sondern ein prozesshaftes Lebensgeschehen, das sich durch bestimmte strukturell-funktionelle (Integrität), regulativ-adaptive (Reagibilität) und kommunikativ-kooperative (Aktivität) Merkmale näher kennzeichnen lässt.

Martin hat psychische Gesundheit definiert als Fähigkeit des Menschen, mit seinem Leben zurechtzukommen, dauerhafte und emotional befriedigende Beziehungen $\mathrm{zu}$ anderen Menschen zu unterhalten, erfolgreich zu arbeiten, entspannen und sich freuen zu können, die eigenen Gaben und Unzulänglichkeiten realistisch einzuschätzen, sich seines eigenen Wertes bewusst zu sein (2).

Von dieser Grundlage her kann man sich dem Verständnis von Gesundheit als einem konkreten Resultat individuellen Lebensvollzugs in der tatsächlichen Lebenswirklichkeit annähern. Leben und Gesundheit sind unteilbar, man hat sie oder man hat sie nicht. Ein allgemeiner Gesundheitsbegriff reflektiert haltlose Vermutungen und weckt falsche Vorstellungen und unzutreffende Erwartungen. Als häufig in der Öffentlichkeit benutzte Redewendung ist diese Gesundheit irrelevant und irrational. Irrelevant, weil beziehungslos, irrational, weil unkonkret unbestimmt.

In der versorgungsmedizinischen Praxis geht es um die Prävention und Behandlung von Beschwerden, Befindensstörungen und Beeinträchtigungen zumeist chronisch Kranker und multimorbider (polypather) Patienten mit der Maßgabe, das individuelle Leistungsvermögen, die Lebensqualität und die Leistungsfähigkeit weitgehend $\mathrm{zu}$ sichern, wiederherzustellen und/oder durch geeignete Maßnahmen auszugleichen. Die Erfüllung und Umsetzung dieses Versorgungsauftrags bedarf notwendig und unerlässlich des Miteinanders von Betroffenen und Beteiligten, einer auf die Persönlichkeit abgestimmten umfassenden ganzheitlichen Sichtweise und nicht zuletzt eine Vorgehens- und Verfahrensweise, die sowohl im Einklang mit der individuellen Lebenssituation und den Lebensumständen als auch in Übereinstimmung mit den gemeinschaftlichen Normen und Werten steht.

In diesem Kontext gewinnt Gesundheit ihren Stellenwert als ständige Herausforderung der eigenen Lebensgestaltung mit einem größtmöglichen Maß des Erhalts an Selbstständigkeit, getragen von bewusst wahrgenommener Selbstverantwortung und begleitet von einem angemessenen Selbstverständnis. Unter dieser Prämisse haben auch Fitness und Wellness ihre Bedeutung, wenn sie nicht missverstanden werden als andauernde Glücksverheißung auf ein langes und unbeschwertes Leben ohne jedwede Anstrengungen oder Bemühungen.

Dr. med. Paul Kokott,

Arzt für Allgemeinmedizin, Salzgitter

\section{Literatur}

1 Wirth A. Adipositas, Ätiologie, Folgeerkrankungen, Diagnostik, Therapie. 3. Auflage. Heidelberg: Springer, 2008

2 Martin B. Psychopathologie. In: Keil W, Sader M (Hrsg.). Grundlagen der Psychologie. München: Juventa 1976 